\title{
Novel Microwave Apparatus for Breast Lesions Detection: Preliminary Clinical Results
}

\author{
Lorenzo Sani ${ }^{\mathrm{a}}$, Navid Ghavami ${ }^{\mathrm{a}, *}$, Alessandro Vispa ${ }^{\mathrm{a}}$, Martina Paolia ${ }^{\mathrm{a}}$, Giovanni Raspa ${ }^{\mathrm{a}}$, Mohammad \\ Ghavami $^{\mathrm{b}}$, Francesco Sacchettic ${ }^{\mathrm{c}}$,Eleonora Vannini ${ }^{\mathrm{d}}$, Simonetta Ercolani ${ }^{\mathrm{d}}$, Andrea Saracini ${ }^{\mathrm{d}}$, \\ Michele Duranti ${ }^{\mathrm{d}}$, Gianluigi Tiberi ${ }^{\mathrm{a}, \mathrm{b}}$ \\ ${ }^{a}$ UBT Umbria Bioengineering Technologies, Spin off of University of Perugia, Perugia, Italy. \\ ${ }^{b}$ School of Engineering, London South Bank University, London, UK. \\ ${ }^{c}$ Physics Department, University of Perugia, Italy. \\ ${ }^{d}$ Department of Diagnostic Imaging, Perugia Hospital, Italy.
}

\begin{abstract}
This paper presents preliminary results of an innovative microwave imaging apparatus for breast lesions detection. Specifically, a Huygens Principle based method is employed to process the microwave signals and to build the respective microwave images. The apparatus has been first tested on phantoms. Next, its performance has been verified through clinical examinations on 22 healthy breasts and on 29 breast having lesions, using as gold standard the output of the radiologist study review obtained using conventional techniques. Specifically, we introduce a metric, which is the ratio between maximum and average of the image intensity (MAX/AVG). We found that MAX/AVG of microwave images can be used for classifying breasts containing lesions. In addition, using MAX/AVG as classification parameter, receiver operating characteristic curves have been empirically determined. Furthermore, for one randomly selected breast having lesion, we have demonstrated that the localization of the inclusion acquired through microwave imaging is compatible with mammography images.
\end{abstract}

Keywords: Microwave imaging, Breast cancer detection, Huygens principle.

\section{Introduction}

Breast cancer is the most common cancer amongst females and the primary cause of death in females of all age groups [1]. Due to this circumstance, mass screening for the purpose of early detection is the way to increase the life expectancy of the patients and to reduce breast cancer mortality rate. At present, screening is achieved through conventional mammogram. National health services are doing all the efforts to enhance mass screening, however it is still insufficient because of many limitations such as the presence of ionizing radiation, the restriction of examination performance to specific conditions, and breast compression. Screening is typically restricted to females between the ages of 45 and 69 years, and it cannot be repeated more than once every two years [2].

Since the early years of the 21st century, microwave imaging has received increasing attention, in particular for its breast cancer detection applications, encouraged by considerable difference between dielectric properties of malignant and normal tissues at microwave frequencies. Specifically, as shown in [3, 4], a fairly significant contrast between healthy breast tissue and malignant tissue is present. This contrast is shown to be up to a factor of 5 in conductivity and permittivity. Meanwhile, newer studies suggest the existence of this contrast only between fatty and malignant breast tissues, and a lower contrast (as low as 10\% in dielectric properties) between healthy fibro glandular and malignant tissues [5-7]. In addition, in [7] it is pointed

${ }^{*}$ Corresponding author 
out that the dielectric properties of benign lesions are similar to the properties of fibro glandular tissues. Younger women's breasts are primarily composed of fibro glandular tissues, with fatty tissues comprising a small percentage of the overall breast volume, hence making them more dense than the breasts of older women [8].

Current ongoing research on microwave breast imaging is separated mainly into microwave tomography and ultra wideband (UWB) radar techniques [5]. Microwave tomography attempts to reconstruct the full electrical profile of the breast by solving a rather ill-posed and nonlinear inverse scattering problem [9, 10]. Complex mathematical formulations causing solution instability is one of the main drawbacks of microwave tomography. On the contrary, radar-based imaging requires the solving of a simpler problem of finding the scattering map based on the differences in dielectric properties of the breast tissues [11], but does suffer from low signal to clutter ratio (S/C). Examples of such imaging methods have been presented in [12, 13].

Recently, an innovative apparatus based on UWB microwaves has been constructed, tested and validated through phantom based measurements $[14,15]$. The apparatus collects the microwave signals, which are then processed using the Huygens Principle (HP). In addition to its simplicity, the method is able to capture the differences and discriminate between varying tissues, or various conditions of tissues, and depict this contrast through the resulting final image. Through gathering the information from the individual frequencies and combining them, HP method permits the utilization of all the frequency domain information, which results in the construction of an image that is consistent. HP algorithm can pinpoint significant scatterer (inclusion) presence and location inside a volume. Validations of the employed method and apparatus on phantoms are shown in this paper. In addition, the capability of the device to detect breast lesions has been verified through clinical examinations on 22 healthy breasts and on 29 breast having lesions (including carcinoma, fibroadenoma, microcalcifications). Specifically, we introduce a metric to measure the non-homogenous behaviour of the image, establishing a modality to detect the presence of a lesion inside a breast.

This paper is organized as follows. In section 2 we describe the hardware of the apparatus, introduce the HP imaging theory and explain the experimental set-up used for both phantom and in-vivo measurements. Section 3 shows the corresponding experimental results, which are then discussed in section 4 . Finally section 5 concludes the paper and addresses future works.

\section{Methodology and experimental configuration}

\subsection{Apparatus and Imaging Procedure}

All microwave images and results shown here were reconstructed from data gathered using our prototype installed at the Department of Diagnostic Imaging, Perugia Hospital, Perugia, Italy.

The apparatus is constituted by one transmitting antenna (denoted here as TX) and by one receiving antenna (here denoted as RX). Both antennas operate in the frequency band of 1 to $9 \mathrm{GHz}$. The apparatus is additionally constituted by: a hub with a cup that are placed to contain the breast of the patient (prone positioned) and two arms to rotatably associate TX and RX to the hub. The hub and the cup were designed after considering the shape and size of the breast. A mature breast has a protruding conical form with a hemispherical shape, having an average diameter between 7 and $8 \mathrm{~cm}$, and weighing between 150 and 225 grams. The transmitting antenna is placed more radially external compared to the receiving antenna. The receiving antenna is placed more radially external with respect to the cup containing the breast (see Fig 1).

Both transmitter and receiver antennas are configured to be rotated around the azimuth, such that they can pick up the reflected electromagnetic field in all the different directions. Both antennas are connected to a Vector Network Analyzer VNA (Copper Mountain Technologies, IN, USA). The apparatus (appropriately integrated in a bed), the VNA and the processing unit can be seen in Fig 2.

To generate the image, the signals measured by the receiving antenna, i.e. the complex S21 output from the VNA, are processed by processing unit through an imaging algorithm based on HP. In this way, a digital image is generated. The apparatus presented here does not require any matching liquid; thus, the antennas and the breast are in free space; the cup, made in Teflon and $1 \mathrm{~mm}$ thick, only has the purpose of better containing the breast. We note that the thickness and the dielectric constant of the Teflon, i.e. 2.1, allow neglecting the effects of the cup for the frequency here considered. 

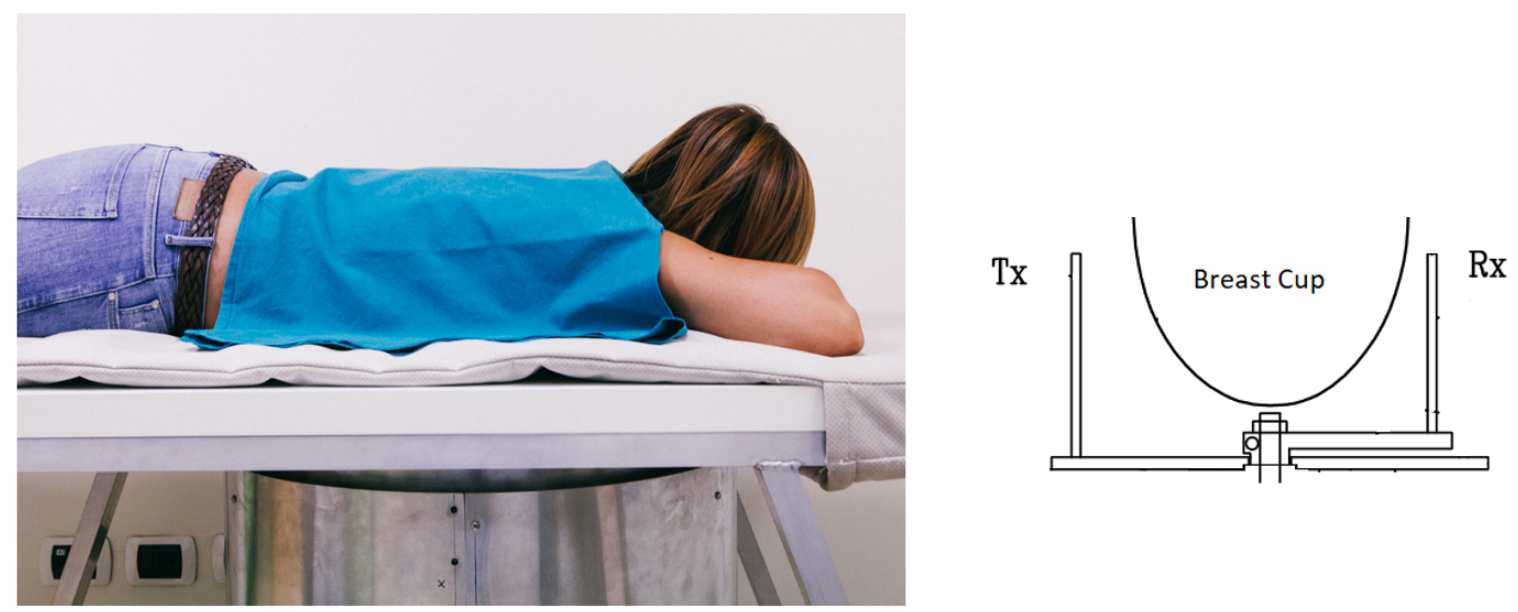

Figure 1: The microwave apparatus consists of a cup that holds the breast when the patient lies prone on the examination table. The transmitting (TX) and receiving (RX) antennas are located inside a hub and can be moved around the azimuth, to irradiate the breast (through TX) and capture the microwaves scattered by the breast itself (through RX). Both TX and RX are connected to a VNA. No matching liquid is required.

To understand how HP works, let us refer to Fig 3, where an object, here a cylinder, in free space is illuminated by a transmitting antenna operating at the frequency $f$. We assume that the received signal is measured at the points $\mathrm{rx}_{n p} \equiv\left(a_{0}, \phi_{n p}\right) \equiv \vec{\rho}_{n p}$, placed along a circular surface with radius $a_{0}$ :

$$
\left.E_{\mathrm{t} x_{m}}^{\mathrm{known}}\right|_{\mathrm{r} x_{n p}}=E_{n p, \mathrm{t} x_{m}}^{\mathrm{known}} \quad \text { with } n p=1, \ldots, N_{\mathrm{PT}}
$$

The HP states that: "Each locus of a wave excites the local matter which re-radiates a secondary wavelets, and all wavelets superpose to a new, resulting wave (the envelope of those wavelets), and so on" [16]. Therefore we consider what would happen if we apply the HP using $E_{n p, t x_{m}}^{\text {known }}$ as locus of a wave.

By resorting to the definition of Huygens Principle, cylinder's inside field is calculated as superposition of the fields radiated by the $N_{\mathrm{PT}}$ observation points of eq. (1):

$$
E_{\mathrm{HP}, 2 \mathrm{D}}^{\mathrm{rcstr}}\left(\rho, \phi ; \mathrm{tx}_{m} ; f\right)=\Delta_{s} \sum_{n p=1}^{N_{P T}} E_{n p, \mathrm{tx}_{m}}^{\mathrm{known}} G\left(k_{1}\left|\vec{\rho}_{n p}-\vec{\rho}\right|\right)
$$

where $\Delta_{s}$ is the spatial sampling, $(\rho, \phi) \equiv \vec{\rho}$ is the observation point and $k_{1}$ represents the wave number for the media internal to the surface where measurements are collected. In eq. (2), the string "rcstr" indicates that the internal field is "reconstructed", and the string "HP,2D" shows that a two-dimensional HP based procedure is used. It can be seen that in eq. (2), we use the Green's function $G$ to propagate the field. Note that, if the conductivity of the media is not equal to zero, eq (2) compensates the attenuation experienced when going into the media. Assuming we use $N_{F}$ frequencies $f_{i}$, and $M$ transmitting sources tx $x_{m}$ with $m=1,2, \ldots, M$, we can determine an image $I$ by incoherent summation of all the solutions:

$$
I(\rho, \phi)=\sum_{m=1}^{M} \sum_{i=1}^{N_{F}}\left|E_{\mathrm{HP}}^{\mathrm{rcstr}}\left(\rho, \phi ; \operatorname{tx}_{m} ; f_{i}\right)\right|^{2}
$$

Such image represents a homogeneity map of the dielectric properties $[17,18]$.

It should be emphasised that the HP procedure does not give the correct internal field, even when dealing with homogenous problems. This is due to the essence of HP, which is formulated for far field phenomena, i.e. it refers to a propagating wavefront. More in detail, to be able to capture the far field, only the knowledge of the $E$ field is enough as we have plane wavefronts. On the other hand, to be able to capture and recover the near field, the knowledge of the $H$ field or normal derivative of the $E$ field is also necessary. Anyhow, 


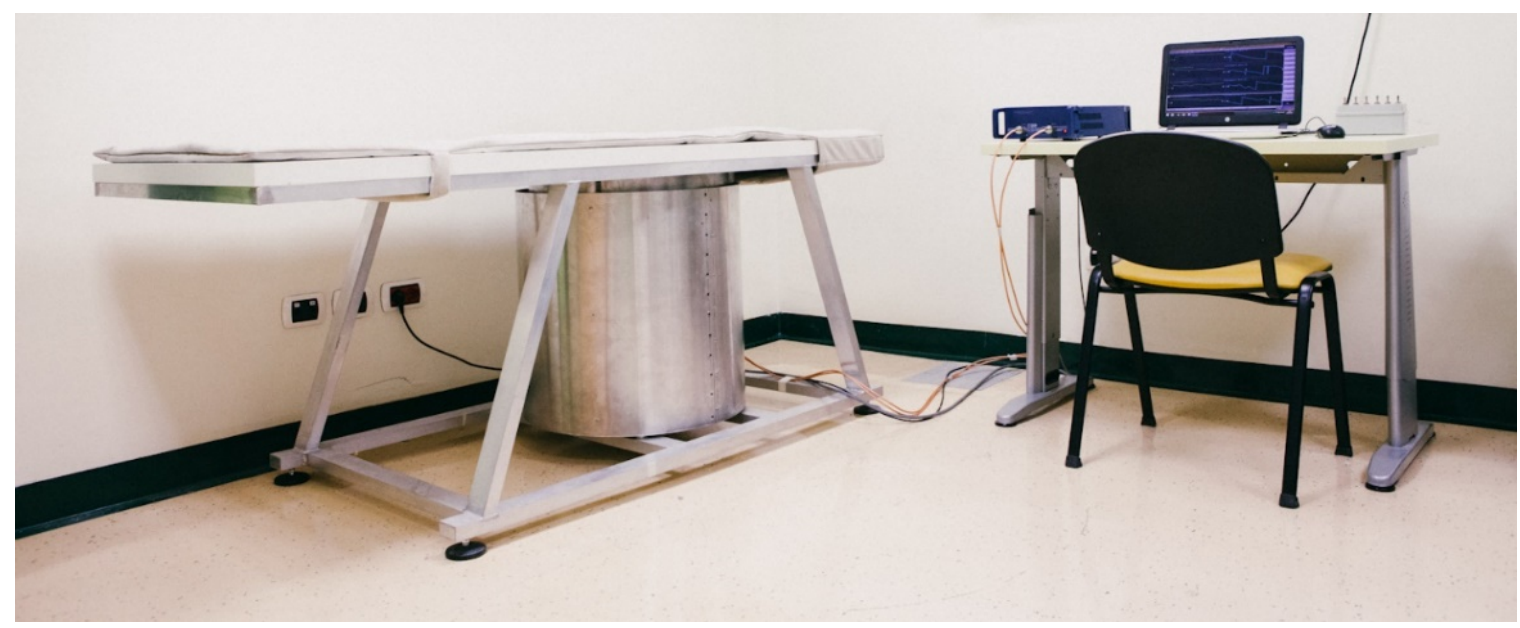

Figure 2: Microwave apparatus.

in this case the intention is not to evaluate the internal field, but to see if the HP can capture the contrast (mismatch boundaries) and locate an inclusion within a volume.

\subsection{Validation in Phantoms}

Prior to in-vivo validation, the first phantom experiment was performed using a cylindrical phantom ( radius $=6 \mathrm{~cm}$, height $=24 \mathrm{~cm}$ ) filled with sunflower oil containing one tube (radius $=0.75 \mathrm{~mm}$, height $=24$ $\mathrm{cm}$ ) filled with water containing $0.1 \mathrm{M} \mathrm{NaCl}$. The tube was eccentrically placed, at a distance of $4.5 \mathrm{~cm}$ from the cylindrical phantom center. Sunflower oil and water containing $0.1 \mathrm{M} \mathrm{NaCl}$ was chosen to guarantee a quite significant contrast (equal or greater than 5) in permittivity and conductivity [19]. To perform the measurements, the cylinder was positioned inside the cup, vertically oriented.

S21 measurements at 1601 frequencies in the range of 1 to $9 \mathrm{GHz}$ were recorded for each transmitting and receiving position, using a frequency step of $5 \mathrm{MHz}$. Transmitter and receiver antennas were placed at the same height; the 15 transmitter positions were separated in 5 groups centred at $0^{\circ}, 72^{\circ}, 144^{\circ}, 216^{\circ}, 288^{\circ}$ on such azimuth plane. Each transmitting group includes 3 transmitting positions distanced $4.5^{\circ}$ from each other. In addition, 80 receiving positions distanced $4.5^{\circ}$ from each other were used. This and all the other measurements described here only used the nominal output power level of the VNA $(1 \mathrm{~mW})$.

We reconstructed the two-dimensional image in the azimuthal plane, i.e. coronal plane, using eq. (3) and processing the 5 transmitting groups separately for artefacts removing [18]. In more details, in [18] it has been shown that the image of the transmitting antenna may mask the inclusion. To remove the image of the transmitting antenna, we employ here the subtraction between the received signal (obtained for a given transmitter position) and the average of the received signals (obtained illuminating the object using the 3 transmitter positions of the correspondent group). We reconstructed the images in a cylindrical grid with radius of $9 \mathrm{~cm}$ (equal to the receiving antenna distance for this experiment), a radial sampling of $1 \mathrm{~mm}$ and a azimuthal sampling of $3^{\circ}$. Due to the position of the receiving antenna in free space and sunflower oil's negligible losses due to conductivity, $k_{1}=k_{0}$ (with $k_{0}$ being free space wave number) was used when achieving the images. Note that, if using $k_{1}=k_{0}$ eq. (2) does not compensate the attenuation experienced when going into the media. Images are normalized to the correspondent maximum.

Next, the experiment was repeated using a cylindrical phantom (radius $=6 \mathrm{~cm}$, height $=24 \mathrm{~cm}$ ) filled with sunflower oil containing two tubes (radius $=0.75 \mathrm{~mm}$, height $=24 \mathrm{~cm}$ ) filled with water containing $0.1 \mathrm{M}$ $\mathrm{NaCl}$. The tubes were eccentrically placed, at a distance of $3.5 \mathrm{~cm}$ and $2.2 \mathrm{~cm}$ from the cylindrical phantom center, respectively.

Finally, to assess the instrument accuracy, a repeatability test was performed in unloaded conditions, i.e. without phantoms. More in details, 10 consecutive S21 measurements were recorded and used to evaluate the average error in S21 measurements, taking the first measurement as reference. 


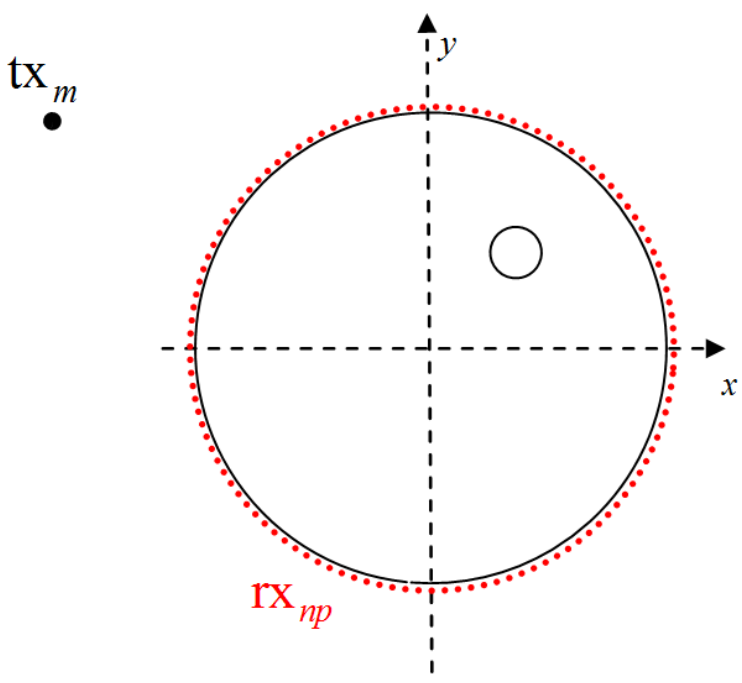

Figure 3: Pictorial view of the problem, with the black and red dots representing the transmitting and receiving positions, respectively.

\subsection{In-vivo Validation}

In 2015, the clinical validation of the microwave apparatus on 45 volunteers was approved by the Ethical Committee of Umbria region, Italy (N. 6845/15/AV/DM of 14/10/2015), which is presently progressing with trials at Perugia Hospital in Italy. Recently, Foligno Hospital, Italy, has been authorized by the Ethical Committee of Regione Umbria, Italy, to join the clinical validation, which has been extended to 100 volunteers (N. 10352/17/NCAV of 16/03/2017). The correspondent protocol focuses on a feasibility study for detection of breast lesions using the proposed microwave mammogram apparatus, with the goal of quantifying the potential of the proposed microwave mammogram apparatus to be used for medical technology screening and possible localization of breast cancer [15].

We present the results of the first 51 breasts, each one with the correspondent output of the radiologist study review obtained using echography and/or mammography and/or MRI and/or histological exam (where required). Echography was performed using the MyLab 70 xvg Ultrasound Scanner (Esaote, Genova, Italy); mammography was performed using Selenia LORAD Mammography System (Hologic, Marlborough, MA); magnetic resonance imaging was performed through a 3.0 T MAGNETOM scanner (Siemens Healthcare, Erlangen, Germany). The correspondent output of the radiologist study review has been used as gold standard for classification of the breasts in two categories: healthy (i.e. breasts with no lesion) and nonhealthy (i.e. breasts containing lesions). In addition, where possible, the breast type has been classified accordingly to its density, following the scale defined by the American College of Radiology (ACR) which goes from ACR1 type A (extremely fatty breast) to ACR type D (extremely heterogeneous fibroglandular breast) [20]. The lesion type, if present, has been classified as carcinoma, fibroadenoma, microcalcifications and other lesions [21-23].

All the subjects have been requested to read and sign both the informative sheet and the informed consent sheet prior to the study. All protocol procedures were in agreement with the institutional and national research committee ethical standards, and with the Helsinki declaration (1964) and its later amendments or comparable ethical standards. The protocol was performed in compliance with the World Medical Association's Code of Ethics regarding human experiments.

After the agreement from the subject to participate, she is assisted by the clinical study coordinator; the subject (prone) positions her breast in the cup that is appropriately integrated in a bed as shown in Fig 2. It is worthwhile pointing out that no matching liquid is used in the apparatus, i.e. antennas and the breast are in free space. The whole process of data acquisition is computer controlled, while a microwave system operator will be present in the room for observation. For each breast, approximately 20 minutes of 
exam data collection will be required. The recorded data is then transferred to a secure server following the exam completion, for image processing. Furthermore, after the exam, to study subject tolerability of the microwave system, a test was administered to all the subjects in order to monitor their comfort.

S21 measurements at 1601 frequencies in the range of 1 to $9 \mathrm{GHz}$ were recorded for each transmitting and receiving position, using a frequency step of $5 \mathrm{MHz}$. Transmitter and receiver antennas were placed at the same azimuthal plane height that crosses the centre of the breast of the prone subject. This was done after making sure the half power beam angle of the antennas include the breast. 15 transmitter positions were separated into 5 groups centred at $0^{\circ}, 72^{\circ}, 144^{\circ}, 216^{\circ}, 288^{\circ}$ on such azimuth plane. Each transmitting group includes 3 transmitting positions distanced $4.5^{\circ}$ from each other. In addition, similar to the phantom experiments 80 receiving positions distanced $4.5^{\circ}$ from each other were used.

Two-dimensional coronal (i.e. azimuth plane) images were reconstructed off-line using eq. (3); artefacts removing was managed as described before. The images have been obtained in a cylindrical grid having radius equal to $7 \mathrm{~cm}$ (which corresponds to the distance of the receiving antenna for this experiment), a radial sampling of $1 \mathrm{~mm}$ and a azimuthal sampling of $3^{\circ}$.

Due to the placement of receiving antenna in the free space and having breast tissues' losses due to conductivity, the images have been obtained using free space dielectric constant and setting the conductivity to $0.4 \mathrm{~S} / \mathrm{m}$. Thus, eq. (2) compensates the attenuation experienced when going into the media.

To be able to perform the comparison between subjects, all intensity images were normalized to unitary average of the intensity. In addition, we introduce a metric to measure the non-homogenous behavior of the microwave image, which is the ratio between maximum and average of the image intensity (MAX/AVG). Using the gold standard output of the radiologist study review, we calculated: the mean and standard deviation of MAX/AVG for the healthy breasts; the mean and standard deviation of MAX/AVG for the nonhealthy breasts. Unpaired t-test for MAX/AVG of the healthy breasts and MAX/AVG of the non-healthy breasts has been also performed. Finally, we empirically evaluated the receiver operating characteristic (ROC) curve using MAX/AVG for classification. Specifically, we evaluated True Positive (TP) rate and False Negative (FN) rate as following:

1. we fix a given Threshold for the parameter MAX/AVG

2. if MAX/AVG $>$ Threshold, the image is classified as positive, i.e. non-healthy

3. if MAX/AVG < Threshold, the image is classified as negative, i.e. healthy

4. we change the value of the Threshold and we repeat the previous steps

ROC curve is determined using TP and FN rate values obtained for the different Thresholds. ROC curve is determined also for the subset of dense breasts, i.e. ACR type $\mathrm{C}+\mathrm{D}$.

\section{Results}

The average error in 10 consecutive S21 measurements, unloaded condition, was $<0.1 \%$. Fig 4 depicts the image corresponding to the phantom with one inclusion, following normalization to its maximum value and after applying a threshold of 0.7 (i.e. converting intensity values lower than 0.7 , to 0 , and ranging values greater than 0.7 from 0 to 1 ). Looking at the figure, we can clearly detect and locate the inclusion, with an error in localization $(<3 \mathrm{~mm})$.

Fig 5 depicts the image corresponding to the phantom with two inclusions, following normalization to its maximum value and forcing the intensity values lower than 0.7 to 0 . From Fig 5, it is again possible to clearly detect and locate both inclusions, again with $<3 \mathrm{~mm}$ error in localization.

In Table 1, we summarize some details on the patient population used for this study; breast density details of the patient population are highlighted in Table 2. Specifically, according to the radiologist study review, 22 healthy breasts (16 dense) and 29 non-healthy breasts (21 dense) underwent microwave imaging (which included 7 carcinoma, 9 fibroadenoma, and 5 microcalcifications). Concerning the clinical images, as an example, microwave images are given for one scattered fibroglandular healthy breast (Fig 6a) and for one heterogeneously dense breast with carcinoma located in the inner quadrants (Fig 7a). Microwave images are homogeneity maps of tissues' dielectric properties, and are given here as $2 \mathrm{D}$ images in the azimuthal 


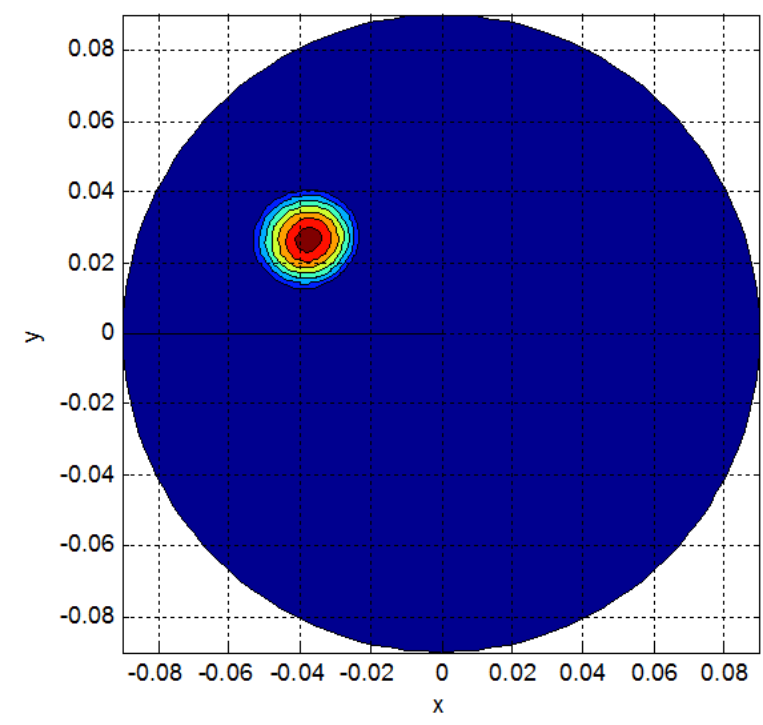

Figure 4: Microwave image obtained for a phantom with a single inclusion, following normalization to its maximum value and forcing the intensity values below 0.7 to 0 . $\mathrm{X}$ and $\mathrm{Y}$ are given in meters.

plane, i.e. coronal plane. Microwave images are normalized to unitary average of the intensity. X and Y axes are given in meters. Intensity is given in arbitrary unit, with a scale from 0 to 2 . A certain level of in-homogeneity can be seen in both Fig 6a and Fig 7a; however, in-homogeneity is more pronounced in Fig 7a. The correspondent mammography images are given in Figs 6b,c and Figs 7b,c. As summarized in Table 3 , for the healthy breasts mean and standard deviation of MAX/AVG are 1.70 and 0.09, respectively; for the non-healthy breasts mean and standard deviation of MAX/AVG are 1.82 and 0.11 , respectively. Unpaired t-test for MAX/AVG of the healthy breasts and MAX/AVG of the non-healthy breasts leads to a $\mathrm{P}$ value of 0.049. Different thresholds for MAX/AVG, spanning from $(1.70-3 \times 0.09)$ to $(1.70+3 \times 0.09)$, have been used to calculate $\mathrm{TP}$ rate and $\mathrm{FN}$ rate, and thus to empirically evaluate the ROC curve. ROC curve is determined also for the subset of dense breasts. ROC curves are given in Fig 8 . We found: $\mathrm{TP}=0.82$ with a corresponding $\mathrm{FN}=0.5 ; \mathrm{TP}=0.7$ with a corresponding $\mathrm{FN}=0.35$. For dense breasts, we found: $\mathrm{TP}=0.95$ with a corresponding $\mathrm{FN}=0.5 ; \mathrm{TP}=0.75$ with a corresponding $\mathrm{FN}=0.35$.

Table 1: Patient population used for this study, with some details including the correspondent output of the radiologist study review.

\begin{tabular}{|c|c|c|c|c|c|c|c|}
\hline & $\begin{array}{c}\text { Total } \\
\text { cases } \\
(\mathrm{n})\end{array}$ & $\begin{array}{c}\text { Mean } \\
\text { age } \\
(y)\end{array}$ & $\begin{array}{c}\text { Age } \\
\text { range } \\
(y)\end{array}$ & Carcinoma & Fibroadenoma & Microcalcifications & $\begin{array}{l}\text { Other } \\
\text { lesions }\end{array}$ \\
\hline Healthy breasts & 22 & 45 & $29-70$ & - & - & - & - \\
\hline Non-healthy breasts & 29 & 52 & $20-86$ & 7 & 9 & 5 & 8 \\
\hline
\end{tabular}

Table 2: Breast densities of the patient population used for this study.

\begin{tabular}{|c|c|c|c|}
\cline { 2 - 4 } \multicolumn{1}{c|}{} & ACR density A + B & ACR density C + D & $\begin{array}{c}\text { ACR density not } \\
\text { available }\end{array}$ \\
\hline Healthy breasts & 3 & 16 & 3 \\
\hline Non-healthy breasts & 3 & 21 & 5 \\
\hline
\end{tabular}

Table 4 shows the list of questions administered to all subjects in order to monitor their comfort; in the same table, the percentages of YES/NO answers are also given. 


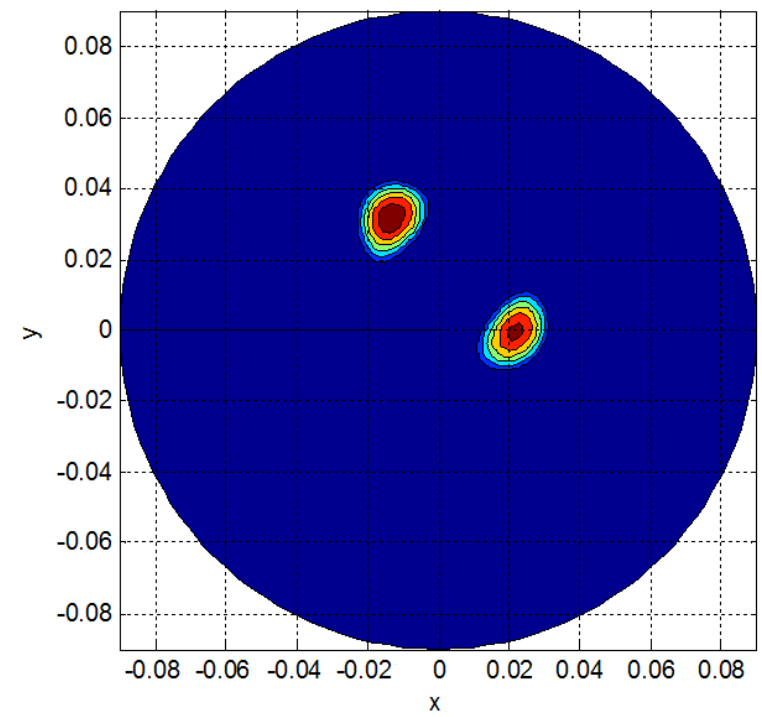

Figure 5: Microwave image obtained for a phantom with multiple inclusions, following normalization to its maximum value and forcing the intensity values below 0.7 to $0 . \mathrm{X}$ and $\mathrm{Y}$ are given in meters.

Table 3: Mean and standard deviation of MAX/AVG of the microwave images for healthy and non-healthy breasts.

\begin{tabular}{|c|c|c|}
\cline { 2 - 3 } \multicolumn{1}{c|}{} & $\begin{array}{c}\text { Mean of Max/Avg of the } \\
\text { microwave images }\end{array}$ & $\begin{array}{c}\text { Standard deviation of Max/Avg of } \\
\text { the microwave images }\end{array}$ \\
\hline Healthy breasts & 1.70 & 0.09 \\
\hline Non-healthy breasts & 1.82 & 0.11 \\
\hline
\end{tabular}

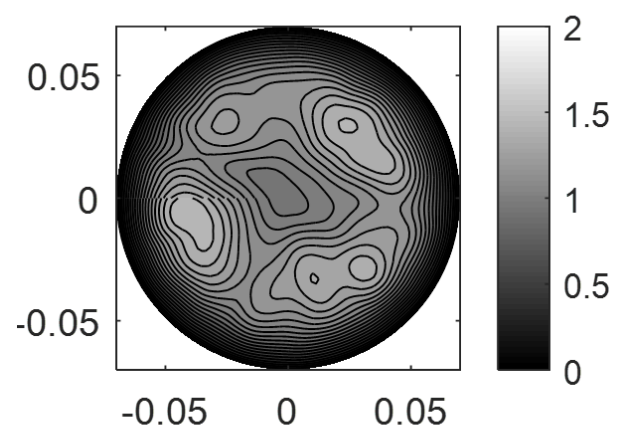

(a)

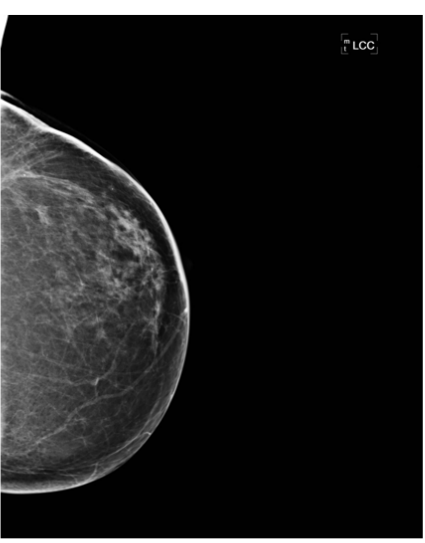

(b)

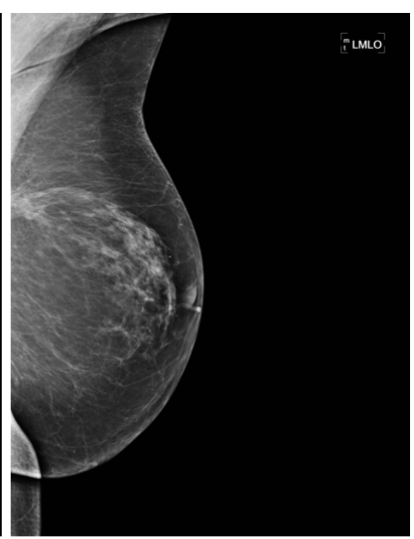

(c)

Figure 6: As an example, a microwave image is given for: (a) scattered fibroglandular healthy breast. The correspondent mammography images are given in $(b, c)$. Microwave images are homogeneity maps of tissues' dielectric properties, and are given here as $2 \mathrm{D}$ images in the azimuthal plane, i.e. coronal plane. Microwave images are normalized to unitary average of the intensity. $\mathrm{X}$ and $\mathrm{Y}$ are given in meters. Intensity is given in arbitrary unit, with a scale from 0 to 2 


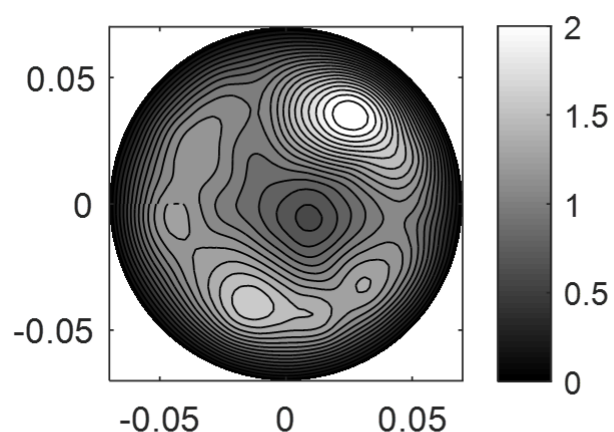

(a)

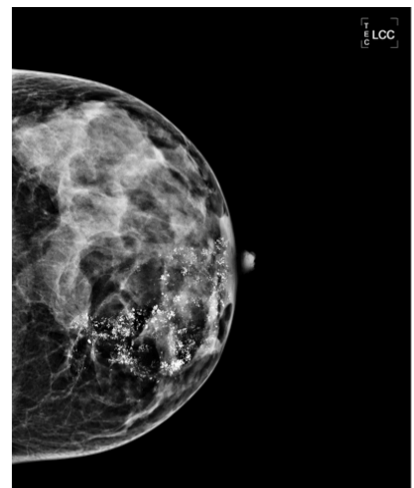

(b)

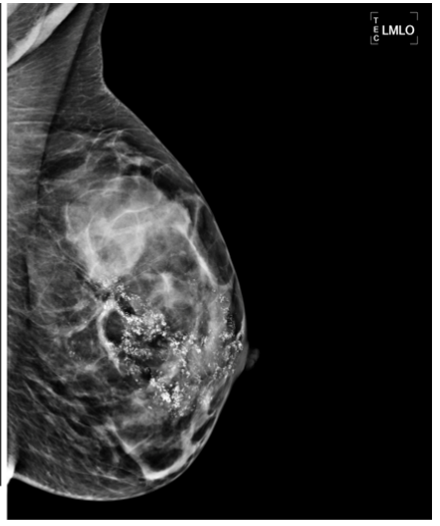

(c)

Figure 7: As an example, a microwave image is given for: (a) heterogeneously dense breast with carcinoma located in the inner quadrants. The correspondent mammography images are given in (b,c). Microwave images are homogeneity maps of tissues' dielectric properties, and are given here as 2D images in the azimuthal plane, i.e. coronal plane. Microwave images are normalized to unitary average of the intensity. $\mathrm{X}$ and $\mathrm{Y}$ are given in meter. Intensity is given in arbitrary unit, with a scale from 0 to 2 .

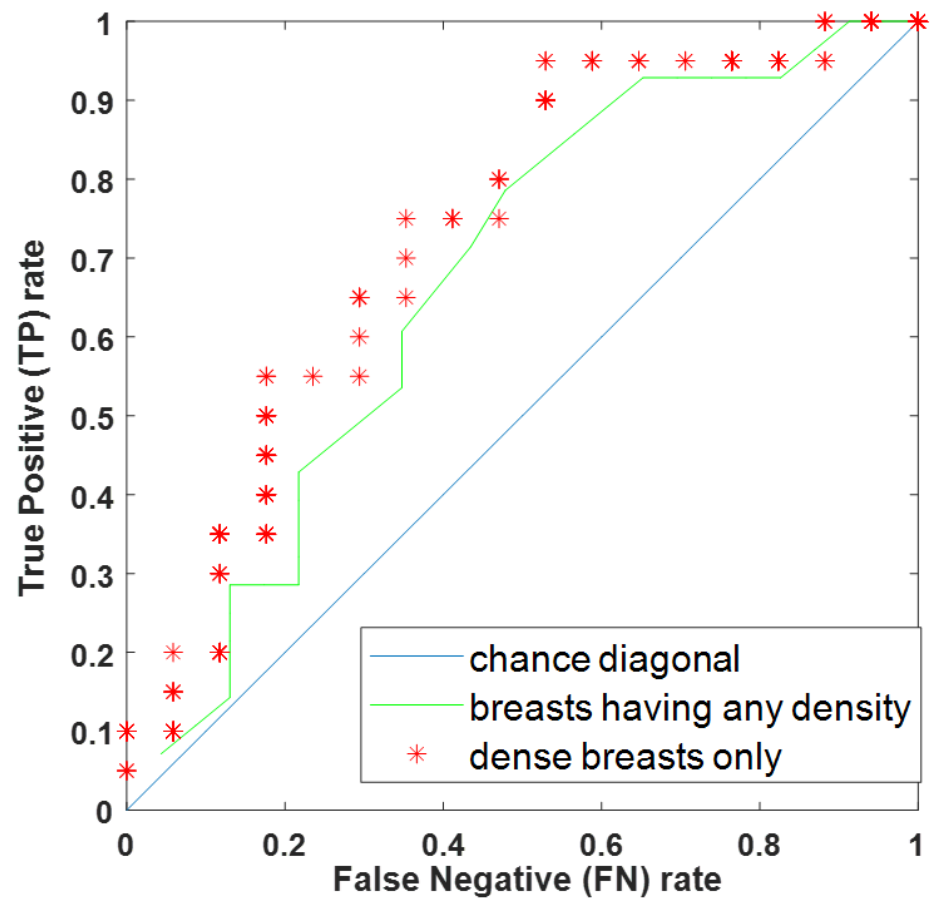

Figure 8: Receiver operating characteristic (ROC) curves for breasts having any density and for dense breasts only. We numerically evaluated the ROC curves using MAX/AVG for classification.

Table 4: List of questions administered to all subjects in order to monitor the comfort of microwave system.

\begin{tabular}{|c|c|c|}
\hline Questions administered to the subjects & YES (\%) & NO (\%) \\
\hline Did you ask for microwave exam interruption? & 0 & 100 \\
\hline Did you find the microwave exam uncomfortable? & 0 & 100 \\
\hline Did you find the microwave exam long lasting? & 50 & 50 \\
\hline
\end{tabular}




\section{Discussions}

The capability of the apparatus to detect and locate an inclusion has been verified through measurements on phantoms. As previously obtained in an anechoic chamber [18], we can also observe here that the final image encounters mismatches within the region of transition of the media, which allows to render the contrast in both conductivity and permittivity.

In the multi-inclusions phantom, both detection and localization are achieved, showing the ability of the methodology in resolving multiple scatterers. In addition, in the multi-inclusions experiment, the two peaks exhibit the same intensity, as the contrast in permittivity and conductivity are the same. The slight error in localization is related to the use of $k_{1}=k_{0}$ in the imaging algorithm; note that this allows to perform imaging even if the dielectric properties of the phantom are unknown [24, 25].

The capability of the device to detect breast lesions has been verified through clinical trials. Specifically, clinical examinations on 22 healthy breasts and on 29 breasts having lesions (including carcinoma, fibroadenoma, and microcalcifications) have been performed, using as gold standard the output of the radiologist study review obtained using conventional examinations. Unlike the procedures such as [26], the proposed procedure does not produce conductivity and permittivity maps in the resulting images; instead images obtained using the proposed procedure are intensity maps representing the homogeneity of tissues' dielectric properties. Microwave images are given here as 2D images in the azimuthal plane, i.e. coronal plane. As the breast is constituted of non-homogeneous tissues, a certain level of non-homogeneity can also be seen in the healthy breast images, as highlighted in Fig 6a; such non-homogeneity will be related to the subjects breast anatomy. However, non-homogeneity is more pronounced in non-heathy breasts, as highlighted in Fig 7a.

A dedicated metric, i.e. MAX/AVG, has been introduced to measure the non-homogenous behaviour of the image, establishing a modality to detect the presence of a lesion inside a breast. Specifically, from Table 4, it is possible to conclude that microwave images of non-healthy breasts have a mean MAX/AVG of approximately $7 \%$ greater than those of the healthy breasts. Unpaired t-test leads to a $\mathrm{P}$ value of 0.049 : by conventional criteria, this difference is considered statistically significant. It follows that MAX/AVG of microwave images can be used for classifying healthy and non-heathy breasts. ROC curves have been empirically determined using MAX/AVG as classification parameter, showing performances which are notinferior to other microwave procedures [27-29]. In addition, conversely to conventional mammography, TP rate increases in the dense breasts; a similar increase has been reported also in [30].

In more details, we obtained $\mathrm{TP}=0.7$ ( $\mathrm{TP}=0.75$ for dense breasts) with a corresponding $\mathrm{FN}=0.35$, values which are not-inferior to [28] where, although, performances have been statistically evaluated using clinical healthy breasts data and injecting simulated tumor response to mimic non-healthy breasts data. A direct comparison with [30] is not straightforward, since in [30] the authors aim to quantify TP having performed microwave imaging in non-healthy breasts only.

Concerning inclusion (i.e lesion) localization, even if a co-registration between Fig 7a and Fig 7b,c is not possible, all figures detect an inclusion near the surface of the breast. Mirror artefacts in microwave images might appear under some particular measurement symmetry circumstances [17]; however, they can be easily removed by breaking the antenna symmetry, where possible. In addition, if a mirror image of the inclusion is displayed in the microwave image, we may refer to the images of the 5 transmitting groups, separately, to discriminate the inclusion from its mirror. In fact, as described in [31], only the true inclusion will generate a doubling in the image. Finally, concerning tolerability of the microwave system, all the subjects found the examination comfortable, even if approximately half of the subjects defined it long lasting; in this context, it is worthwhile pointing out that the examination does not require any breast compression. None of the subjects asked for examination interruption.

\section{Conclusions}

A new microwave imaging apparatus based on HP has been first validated through phantom measurements. It has been shown that HP procedure is robust and permits, in the presence of a contrast in dielectric properties, the detection of inclusions within an object even if the dielectric properties of the object are unknown. In addition, the capability of the device to detect breast lesions has been verified through clinical 
trials on 22 healthy breasts and on 29 breasts having lesions (including carcinoma, fibroadenoma, and microcalcifications). The proposed apparatus is completely safe for both patients and operators, as it does not emit any ionizing radiation and the power employed in microwave imaging is the nominal output power level of the VNA $(1 \mathrm{~mW})$. Moreover, the apparatus does not require any breast compression. We found that MAX/AVG of microwave images can be used for classifying breasts containing lesions. Specifically, using MAX/AVG as classification parameter, ROC curves have been empirically determined. We found $\mathrm{TP}=0.7$ with a corresponding $\mathrm{FN}=0.35$; also, $\mathrm{TP}$ rate increases in the dense breasts. Research is in progress to statistically evaluate ROC curves and to find the parameters, which maximize classification performances.

\section{Acknowledgments}

This project has received funding from the European Unions Horizon 2020 research and innovation programme under grant agreement No 830265. The authors would like to thank Marzia Maccheroni, Department of Diagnostic Imaging, Perugia Hospital, Italy.

\section{References}

[1] E. Petracci, A. Decarli, C. Schairer, et al, "Risk factor modification and projections of absolute breast cancer risk", J Natl Cancer Inst 2011; 103:1037-1048.

[2] http://www.osservatorionazionalescreening.it/.

[3] X. Li and S.C. Hagness, "A confocal microwave imaging algorithm for breast cancer detection", IEEE Microwave Wireless Comp. Lett., vol. 11, pp. 130-132, Mar. 2001.

[4] E.J. Bond, X. Li, S.C. Hagness, B.D. Van Veen, "Microwave imaging via space-time beamforming for early detection of breast cancer", IEEE Transactions on Antennas and Propagation, Vol.: 51 , No. 8, Year: 2003 , pp 1690 - 1705.

[5] N.K. Nikolova, "Microwave Imaging for Breast Cancer", IEEE Microwave Magazine, vol.12, no.7, pp.78-94, Dec. 2011.

[6] M. Lazebnik, L. McCartney, D. Popovic, C.B. Watkins, M.J. Lindstrom, J. Harter, S. Sewall, A. Magliocco, J.H. Booske, M. Okoniewski , et al (2007a), "A large-scale study of the ultrawideband microwave dielectric properties of normal breast tissue obtained from reduction surgeries", Physics in medicine and biology 52(10):2637.

[7] M. Lazebnik, D. Popovic, L. McCartney, C.B. Watkins, M.J. Lindstrom, J. Harter, S. Sewall, T. Ogilvie, A. Magliocco, T.M. Breslin et al (2007b), "A large-scale study of the ultrawideband microwave dielectric properties of normal, benign and malignant breast tissues obtained from cancer surgeries", Physics in Medicine and Biology 52(20):6093.

[8] R. Conceio, J. Mohr, M. O'Halloran, An Introduction to Microwave Imaging for Breast Cancer Detection, Biological and Medical Physics, Biomedical Engineering, Springer, 2016

[9] S.P. Poplack et al, "Electromagnetic breast imaging: Results of a pilot study in women with abnormal mammograms", Radiology, vol. 243,pp. 350-359, 2007.

[10] T. Grzegorczyk, "Fast 3-D tomographic microwave imaging for breast cancer detection", IEEE Trans. Med. Imag., vol. 31, no. 8, pp. 1584-1592, Aug. 2012.

[11] D. Gibbins et al. , "A comparison of a wide-slot and a stacked patch antenna for the purpose of breast cancer detection", IEEE Trans. Antennas Propag., vol. 58, no. 3, pp. 665-674, Mar. 2010.

[12] J. Bourqui et al., "A prototype system for measuring microwave frequency reflections from the breast". Int. J. Biomed. Imag. , vol. 2012, pp. 851234-1-851234-12, 2012.

[13] M. Klemm et al., "Microwave radar-based differential breast cancer imaging: Imaging in homogeneous breast phantoms and low contrast scenarios", IEEE Trans. Antennas Propag. , vol. 58, no. 7, pp. 2337-2344, Jul. 2010.

[14] G. Tiberi, R. Raspa, Apparatus for testing the integrity of mammary tissues, patent n. 0001413526.

[15] L. Sani et al., "Initial Clinical Validation of a Novel Microwave Apparatus for Testing Breast Integrity," 2016 IEEE International Conference on Imaging Systems and Techniques (IST), Chania, 2016, pp. 278-282.

[16] P. Enders, "Huygens principle as universal model of propagation," Latin Amer. J. Phys. Educ., vol. 3, no. 1, pp. 1932, Jan. 2009

[17] G. Tiberi, N. Ghavami, D.J. Edwards, A. Monorchio, "UWB Microwave Imaging of Cylindrical Objects with Inclusions", IET Microwaves, Antennas \& Propagation, Volume: 5, Issue: 12 Publication Year: 2011 , Page(s): 1440 - 1446.

[18] N. Ghavami, G. Tiberi, D.J. Edwards, A. Monorchio, "UWB Microwave Imaging of Objects With Canonical Shape", IEEE Transactions on Antennas and Propagation, Volume: 60 , Issue: 1, Publication Year: 2012 , Page(s): $231-239$.

[19] G. Tiberi et al, "Electromagnetic characterization of an MR volume coil with multilayered cylindrical load using a 2-D analytical approach", Journal of Magnetic Resonance, Volume 230, May 2013, pp. 186197.

[20] American College of Radiology. Practice guidelines and technical standards. Reston, Va: American College of Radiology; 2008. ACR practice guideline for the performance of screening and diagnostic mammography.

[21] F.A. Tavassoli, P. Devilee editors. World Health classification of Tumours. Pathology and genetics of tumours of the breast and female genital organs. IARC press, Lyon 2003.

[22] S. Lakhani, I.O. Ellis, S.J. Schnitt et al, editors. WHO classification of tumour of the breast, 4th edition. IARC: Lyon 2012. 
[23] N. Perry, M. Broeders, C. de Wolf, S. Trnberg, R. Holland, L. von Karsa. European guidelines for quality assurance in breast cancer screening and diagnosis. Fourth Edition. European Commission 2006.

[24] N. Ghavami, G. Tiberi, D. J. Edwards, A. Safaai-Jazi, and A. Monorchio, "Huygens Principle Based Imaging of Multilayered Objects with Inclusions," Progress In Electromagnetics Research B, Vol. 58, 139-149, 2014.

[25] N. Ghavami, P. Probert Smith, G. Tiberi, D. Edwards and I. Craddock, "Non-iterative beamforming based on Huygens principle for multistatic ultrawide band radar: application to breast imaging," in IET Microwaves, Antennas \& Propagation, vol. 9, no. 12, pp. 1233-1240, 2015.

[26] P. M. Meaney, M. W. Fanning, T. Raynolds et al, "Initial Clinical Experience with Microwave Breast Imaging in Women with Normal Mammography", Acad Radiol. 2007 Feb; 14(2): 207-218. doi: 10.1016/j.acra.2006.10.016.

[27] E. C. Fear, J. Bourqui, C. Curtis, D. Mew, B. Docktor and C. Romano, "Microwave Breast Imaging With a Monostatic Radar-Based System: A Study of Application to Patients," in IEEE Transactions on Microwave Theory and Techniques, vol. 61, no. 5, pp. 2119-2128, May 2013.

[28] M. Elahi, D. OLoughlin, B. Lavoie, M. Glavin, E. Jones, E. Fear, M. OHalloran, "Evaluation of Image Reconstruction Algorithms for Confocal Microwave Imaging: Application to Patient Data"'. Sensors 2018, 18, 1678.

[29] Y. Li, E. Porter, A. Santorelli, M. Popovi, M. Coates, "Microwave breast cancer detection via cost-sensitive ensemble classifiers: Phantom and patient investigation," Biomedical Signal Processing and Control, Vol. 31, pp. 366-376, 2017.

[30] A. W. Preece, I. Craddock, M. Shere, L. Jones, and H. L. Winton "MARIA M4: clinical evaluation of a prototype ultrawideband radar scanner for breast cancer detection," Journal of Medical Imaging, 3(3), 033502, 2016.

[31] D. Byrne, I. J. Craddock, "Time-Domain Wideband Adaptive Beamforming for Radar Breast Imaging", IEEE Transactions on Antennas and Propagation, Volume:63, Issue: 4, 2015, 1725 - 1735. 